Check for updates

Cite this: RSC Adv., 2017, 7, 29149

\title{
Mussel-inspired chemistry for preparation of superhydrophobic surfaces on porous substrates
}

\author{
Kaili Wang, ${ }^{\text {ab }}$ Youming Dong, ${ }^{\text {ab }}$ Yutao Yan, ${ }^{\text {ab }}$ Shifeng Zhang (D)*ab and Jianzhang Li ${ }^{* a b}$
}

A facile and versatile mussel-inspired surface modification approach was used to modify porous materials (wood, sponge and stainless steel mesh) to fabricate a superhydrophobic surface. The as-formed polydopamine (PDA) coating can tightly adhere on the porous structure surface, which also provides a versatile platform for secondary reactions to anchor hydrophobic long-chain groups for hierarchical superhydrophobic surfaces preparation. The as-prepared surfaces showed excellent superhydrophobicity with a water contact angle (CA) of about $153^{\circ}$, even after being subjected to harsh conditions, including strong acid-base and organic solvent immersion, high-temperature boiling, ultrasonic washing, and ultraviolet aging. The produced superhydrophobic sponge exhibited an oil absorption capacity of 73-156 times its own weight for a series of oils and organic solvents and showed good recyclability. The obtained stainless steel mesh also presented good oil-water separation ability. Importantly, this modification method provides an efficient, versatile, easy, and mild route to prepare superhydrophobic surfaces for various porous substrates, resulting in a wide range of potential applications.

Received 28th April 2017 Accepted 30th May 2017

DOI: $10.1039 / \mathrm{c} 7 \mathrm{ra0} 4790 \mathrm{~h}$

rsc.li/rsc-advances focused on one kind of small-scale substrate, limiting practical application. ${ }^{7}$

Unlike the above methods, the mussel-inspired multifunctional surface modification has attracted attentions as a green, mild, highly efficient, and sustainable approach. ${ }^{8}$ It was found that the amazing adhesive ability of mussels is conferred by the L-3,4-dihydroxyphenylalanine (DOPA), which allows the mussels to adhere to many types of substrates. Dopamine, a molecular structural mimic of DOPA, can self-polymerize and deposit on material surfaces under a mildly alkaline and aerobic environment, and the as-layer of polydopamine (PDA) plays as an extremely versatile platform for a diverse range of secondary reactions, including the reduction of several noble metallic ions into metallic nanoparticles or reaction with amino and thiol groups through Michael addition or Schiff base reactions. ${ }^{9}$ Therefore, there is reason to believe that a series of functional components can be linked onto the substrate surface, allowing the designs of hierarchical structures to prepare superhydrophobic surfaces.

Wood and other lignocellulosic materials exhibit significant heterogeneity and porously structural complexity. ${ }^{10}$ A superhydrophobic wood surface can minimize the negative effects by water invading, such as dimensional instability or microorganisms/insects' damages. However, traditional approaches for superhydrophobic surfaces on wood and other lignocellulosic materials are complicated, small-scale, and usually generate microstructural damages. For example, hydrothermal or solution-immersed methods may weaken the wood components due to the high temperature and acid-based toxic solvents. ${ }^{11,12}$ Chemical etching and plasma etching require
${ }^{a}$ Key Laboratory of Wood-Based Materials Science and Utilization, College of Materials Science and Technology, Beijing Forestry University, No. 35 Tsinghua East Road, Haidian District, Beijing 100083, P. R. China. E-mail: shifeng.zhang@bjfu.edu.cn; lijzh@bjfu.edu.cn; Fax: +86010 62336072; Tel: +8601062336072

${ }^{b}$ Beijing Key Laboratory of Wood Science and Engineering, College of Materials Science and Technology, Beijing Forestry University, No. 35 Tsinghua East Road, Haidian District, Beijing 100083, P. R. China 
specialized equipment and reagents. ${ }^{13}$ The sol-gel method requires complicated procedures and chemical vapor deposition requires harsh conditions. ${ }^{\mathbf{1 4 , 1 5}}$ In addition, the wood surfaces morphologies are altered by section orientations, where the longitudinal surface is micro-grooved while the transverse surface shows honeycomb-like appearance. Therefore, the superhydrophobicity on the wood longitudinal surface to overcome the naturally micro-grooved structure becomes a challenge. However, the mussel-inspired surface chemistry on sophisticated porous structures can address the problems accordingly.

In this paper, we selected wood, sponge, and stainless steel mesh as three different kinds of porous substances to facilely prepare the superhydrophobic materials by mussel-inspired functionalization approach. Sponge is of high absorption ability, good elasticity and low density. While, stainless steel mesh owns uniform, dense, and monolayer holes. First, dopamine spontaneously self-polymerizes into PDA in a basic environment, and tightly adheres onto the substrate surfaces. Then, the reduced Ag nanoparticles and clusters, along with the PDA nanoparticles create the micro/nano hierarchical roughness. Finally, grafting long-chain alkyl onto as-formed rough surfaces, and the superhydrophobic surfaces were successfully prepared. The mussel-inspired functionalization method has the following innovation points and significant advantages. (1) The selfpolymerization of dopamine can fully apply on a wide range of materials surfaces, and there are no limits on the type or shape of substrates. (2) The PDA layer can act as stable bridges or joints between the substrate and hydrophobic long-chain groups, even play reducibility on $\mathrm{Ag}$ particles, along with the PDA nanoparticles to create the hierarchical roughness. (3) The whole procedure was conducted under mild conditions without intricate instruments. (4) Water and ethanol were used as solvents during the preparation process, avoiding damage to the intrinsic structure and component of substrates. The superhydrophobic surfaces prepared in this study showed outstanding stability and durability in various harsh environments, including strong acid, strong base, organic solvent, high temperature and humidity, ultrasonic washing, and UV radiation.

\section{Experimental}

\section{Materials}

Defect-free and straight-grained sapwood of fast-growing poplar (Populus tomentosa Carr.) was obtained from a wood product manufacturer (Henan, China). The wood samples were cut into $20 \times 20 \times 10 \mathrm{~mm}^{3}$ and $3 \times 20 \times 20 \mathrm{~mm}^{3}$ (radial $\times$ tangential $\times$ longitudinal) blocks. The melamine sponge and stainless steel mesh were purchased from a local market. Dopamine hydrochloride (99\% purity), tris(hydroxymethyl)aminomethane (Tris, 99\% purity), octadecylamine (99\% purity), and octadecanethiol (97\% purity) were purchased from Tianjin Heowns Biochem Co., Ltd. $\mathrm{AgNO}_{3}$ (99.8\% purity) was purchased from Guangzhou Jinhauda Chemical Co., Ltd. Anhydrous ethanol, toluene, acetone, hydrochloric acid, sodium hydroxide, $n$-hexane, $N, N$ dimethylformamide (DMF), dodecane, dichloromethane, trichloromethane, diethylether, styrene, and xylene were purchased from Beijing Chemical Works (China). Sunflower oil and pump oil were purchased from a local market. All chemical reagents were used without further purification.

\section{Preparation of superhydrophobic surfaces on the porous materials}

All wood samples were Soxhlet-extracted with a mixture of toluene/ethanol/acetone $(4: 1: 1 \mathrm{v} / \mathrm{v} / \mathrm{v})$ for $12 \mathrm{~h}$. The samples were thoroughly dried in an oven at $(103 \pm 2)^{\circ} \mathrm{C}$ until a constant weight was reached. The immersion solution $\left(2.0 \mathrm{mg} \mathrm{mL}^{-1}\right)$ was pre-prepared by dissolving dopamine in Tris-HCl (10 $\mathrm{mM})$ buffer solution with $\mathrm{pH}$ 8.5. The wood samples were immersed into the solution and stirred for $24 \mathrm{~h}$ at ambient temperature, allowing deposit of a PDA layer on the surface of the wood blocks. The as-obtained wood samples were washed with deionized water several times, dried in an oven at $60{ }^{\circ} \mathrm{C}$, and labelled as PDA/wood. The PDA/wood samples were placed into $\mathrm{AgNO}_{3}$ solution $\left(1.0 \mathrm{mg} \mathrm{mL}{ }^{-1}\right)$ for $12 \mathrm{~h}$. $\mathrm{Ag}$ nanoparticles were deposited on the PDA-coated surfaces by reduction. These wood samples were then washed with deionized water and dried at $60{ }^{\circ} \mathrm{C}$, and were then labelled as PDA/Ag/wood. The PDA/Ag/ wood samples were put into octadecylamine ethanol solution $(1 \mathrm{v}: 100 \mathrm{v})$ to react at $30^{\circ} \mathrm{C}$ for $24 \mathrm{~h}$, the samples were removed, washed with ethanol several times, and dried at $60{ }^{\circ} \mathrm{C}$ to prepare the superhydrophobic wood. For comparison, another kind of superhydrophobic wood treated with octadecanethiol instead of octadecylamine was also prepared using the above method, and was labelled as PDA/Ag/SH/wood and PDA/Ag/NH/wood, respectively. The same method and procedures were used to modify sponge and stainless steel mesh.

\section{Characterization}

Scanning electron microscopy (FE-SEM, Hitachi S-4800) was performed with an accelerating voltage of $15 \mathrm{kV}$ to observe the surface morphology of the specimens.

Atomic force microscopy (AFM, Bruker Multimode 8) was used to observe the morphology of the superhydrophobic surface. The topographic (height) and phase images were collected in the tapping mode using a monolithic Si tip.

$\mathrm{X}$-ray photoelectron spectroscopy (XPS) was recorded with a K-Alpha X-ray photoelectron spectrometer (Thermo Fisher Scientific Co., Ltd) at room temperature with monochromatic $\mathrm{Al}$ $\mathrm{K} \alpha$ radiation $(1486.6 \mathrm{eV})$.

The superhydrophobic wood surfaces were milled to a 200 mesh particle size and embedded into potassium bromide $(\mathrm{KBr})$ pellets at a weight ratio of $1: 70$. The pellets were then analyzed with an FTIR device (Nicolet 6700 Thermo Scientific, USA) ranging from 4000 to $400 \mathrm{~cm}^{-1}$ at a $4 \mathrm{~cm}^{-1}$ resolution for 32 scans.

Contact angles (CAs) were measured with a $3 \mu \mathrm{L}$ deionized water droplet on a Dataphysics OCA 20 (Dataphysics, Germany) instrument at room temperature. The contact angles were determined by averaging the values obtained at six different points on each sample surface. Sliding angles (SAs) were determined by the minimum tilt angle of the sample platform at which a water droplet $(10 \mu \mathrm{L})$ rolls off the surface. 
Self-cleaning property. Water was poured with a syringe onto a surface contaminated by carbon-powder to compare the selfcleaning properties of the control and superhydrophobic wood samples.

Mirror-like phenomenon test. The superhydrophobic wood sample was immersed into water to visualize mirror-like phenomena.

Chemical durability test. The samples were immersed into $\mathrm{HCl}$ solution $(\mathrm{pH}=2)$, $\mathrm{NaOH}$ solution $(\mathrm{pH}=12)$, and individual organic solvents for $24 \mathrm{~h}$, then the CAs were measured to determine the samples' chemical durability.

Ultrasonic washing test. We examined the washing durability of the superhydrophobic wood by submerging the samples in a volume of distilled water six times for a total of $1 \mathrm{~h}$ under ultrasonication ( $40 \mathrm{kHz}$ frequency, $100 \mathrm{~W}$ ). The samples were collected at certain intervals and dried at $103 \pm 2{ }^{\circ} \mathrm{C}$ for $3 \mathrm{~h}$ followed by contact angle (CA) measurements.

$\mathrm{UV}$ radiation test. The superhydrophobic wood was placed in an ultraviolet aging test chamber (Beijing Beifang Lihui Instrument Equipment. Co., LTD, China) for a week. The UV lamp power was $40 \mathrm{~W}$ and radiation wavelength was $340 \mathrm{~nm}$, and the distance from the samples to the UV lamps was about $10 \mathrm{~cm}$.

Absorption tests of oils/organic solvents. A series of oils/ organic solvents (including $n$-hexane, dodecane, dichloromethane, trichloromethane, diethylether, styrene, xylene, sunflower oil, and pump oil) were tested in the typical absorption process. Briefly, a piece of superhydrophobic sponge was immersed into the oil/organic mixture until it was fully saturated. Next, the saturated sponge was removed and rapidly weighed. The absorption capacity of the superhydrophobic sponge was defined as $\left(M_{1}-M_{0}\right) / M_{0}$, where $M_{0}$ represents the weight of the pristine dry sponge and $M_{1}$ represents the total weight after saturation with liquids.
Oil/water separation with superhydrophobic mesh. selfmade equipment was used to assay oil/water separation with the mixture of water and trichloromethane $(1 \mathrm{v}: 2 \mathrm{v})$. The flux was determined according to the equation: flux $=V / S T$, where $V$ is the volume of filtrates, $S$ is the area of the mesh, and $T$ is the filtration time.

\section{Results and discussion}

\section{Preparation process and reaction mechanism}

A typical procedure of preparing the bionic superhydrophobic surface is shown in Scheme 1. In a basic environment, dopamine spontaneously self-polymerizes into PDA, and tightly adheres on the substrate by strong covalent and noncovalent interactions. The micro/nano hierarchical structure on the PDA coating is then formed by reducing Ag nanoparticles and clusters duo to the great reducing capacity of the catechol groups in PDA. Finally, the grafting of NH-terminated or/and HSterminated long-chain alkyl onto PDA and Ag particles occurs through the Michael-addition and Schiff-base reactions, and metal-thiol coordination, resulting in the hierarchical superhydrophobic surface. Hydrothermal approach is also a method to prepare superhydrophobic surfaces by incorporating inorganic nanoparticles and organic long-chain groups. Nevertheless, that process is complicated, and not suitable for large-area fabrication. In addition, it damages the wood substrates components and structures due to the harsh environments. ${ }^{\mathbf{1 1 , 1 6}}$ On the other hand, the coating methods apparently can realize a large-area superhydrophobicity on substrates surfaces, but reduced CAs were ever reported during the service of superhydrophobic coatings under outdoor exposure, because of the degradation of the surfaces or organic contaminants under environmental conditions, exhibiting poor durability. ${ }^{17,18}$ In the present study, the whole procedure of mussel-inspired

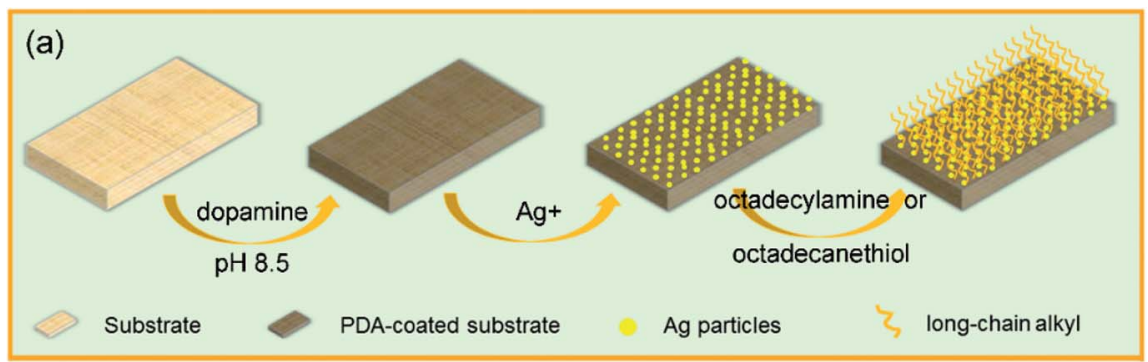

(b)

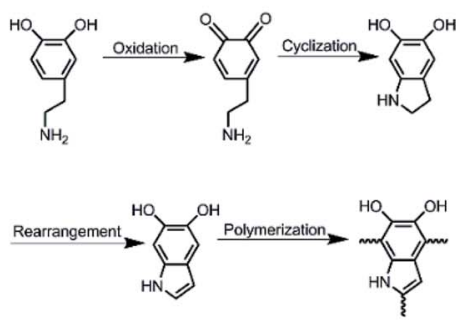

(c)

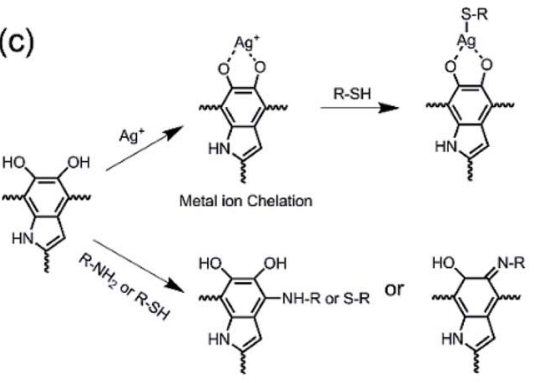


multifunctional surface modification was conducted under mild conditions, avoiding damage to the intrinsic structures and components of wood substrates. And the as-formed superhydrophobic surfaces showed excellent stability and durability in various harsh environments due to the strong interfacial interaction.

\section{Micromorphology and chemical composition analysis}

The surface morphologies of the control and treated wood samples are shown in Fig. 1. The longitudinal and transverse surfaces with different magnifications are shown from left to right. The control wood presents a highly textured cellular structure with a smooth lumen surface. The longitudinally arranged cells constitute the wood surface and feature concave cell lumens and projected cell walls, forming an alternating "valley" and "ridge" structure for the roughness of wood surfaces at the microscale level (Fig. 1a). After the wood samples were immersed in dopamine solutions for $24 \mathrm{~h}$, a thin and much rougher PDA layer was observed on the lumen surface, with aggregated PDA particle diameters in the range of dozens to hundreds nanometers (Fig. 1b). The strong adhesion and reactivity of PDA allowed preparation of a hierarchical surface by depositing Ag nanoparticles and clusters on the surface by dipping the substrate in $\mathrm{AgNO}_{3}$ solution due to the great reducing capacity of the catechol groups in the PDA. Separate Ag nanoparticles and aggregates from tens of nanometers to several micrometers in size were observed on the PDA-coated surface after reduction. The similar approach has been reported for reducing $\mathrm{Ag}$ ion to $\mathrm{Ag}$ nanoparticles on PDA-coated surfaces, which demonstrated the excellent adhesion and stability. ${ }^{19}$ The PDA assemblies and Ag nanoparticles and clusters served as building blocks of the micro/nano multiscale hierarchical structure and accordingly generated high surface roughness on wood substrates (Fig. 1c). After grafting longchain alkyl groups, the superhydrophobic surfaces were successfully prepared (Fig. 1d and e).

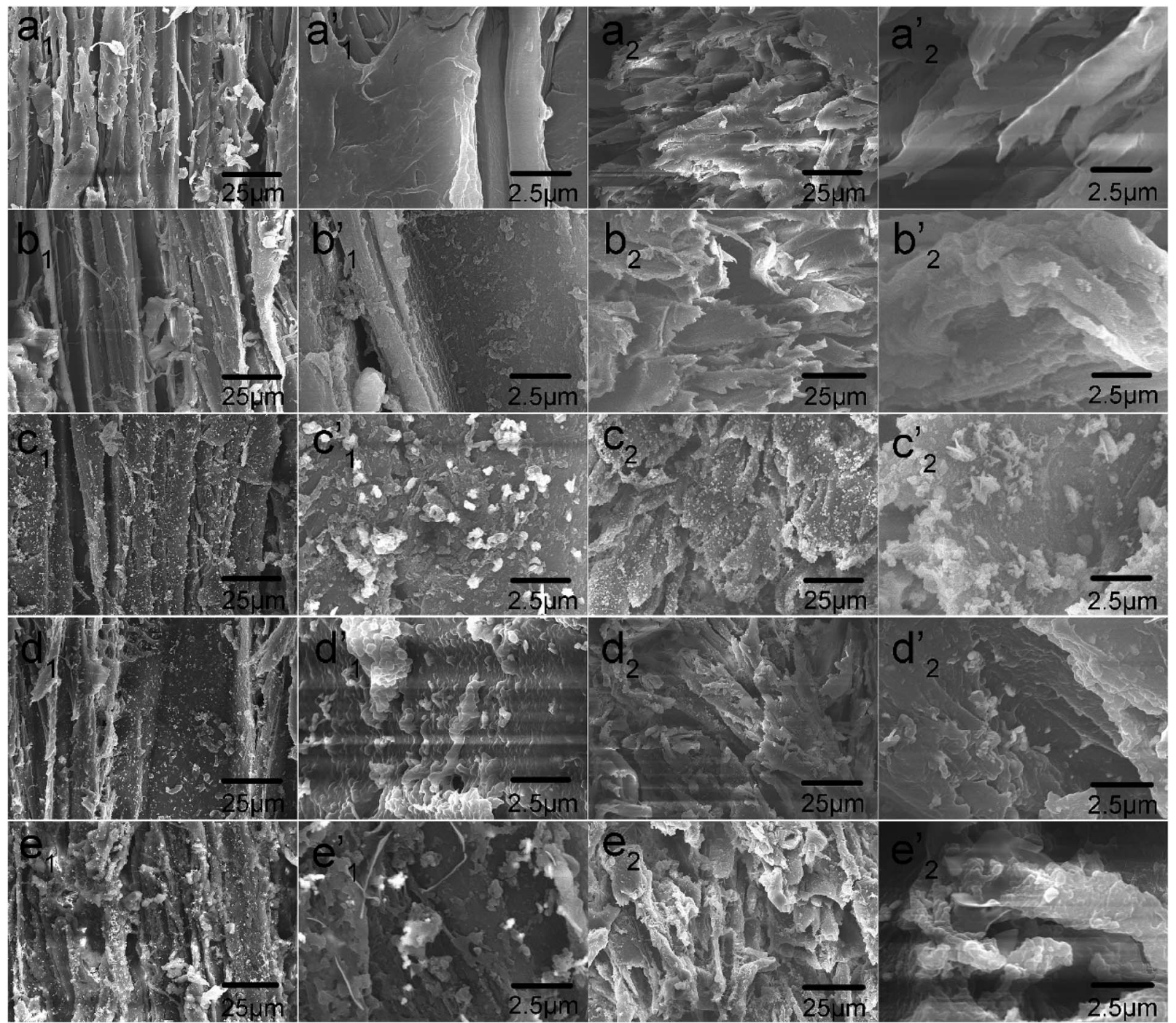

Fig. 1 FE-SEM observations of tangential sections and cross sections of (a) control wood, (b) PDA/wood, (c) PDA/Ag/wood, (d) PDA/Ag/NH/ wood, and (e) PDA/Ag/SH/wood. 
The surface topographies and roughness of the control and treated wood were further examined by AFM. The threedimensional surface plots $(250 \mathrm{~nm} \times 250 \mathrm{~nm})$ AFM images are shown in Fig. 2. The pristine saw-cutting surface with torn fibers is shown in Fig. 2a, with a root-mean-square roughness $\left(R_{\mathrm{q}}\right)$ of $20.6 \mathrm{~nm}$. The PDA/wood exhibited a fine microstructure and consisted of small and uniform grains forming a more complex surface topography with a $R_{\mathrm{q}}$ of $16.8 \mathrm{~nm}$, likely because the PDA nanoparticles were uniformly spread out and filled the grooved wood surfaces (Fig. 2b). With incorporation of the reduced $\mathrm{Ag}$ particles, the well-developed hierarchical structure was formed and the hybrid-coated surface was more rough, with an $R_{\mathrm{q}}$ value of $68.6 \mathrm{~nm}$ (Fig. 2c). The $R_{\mathrm{q}}$ increased to $106 \mathrm{~nm}$ and $95 \mathrm{~nm}$ after grafting of the long chain alkyl groups onto the PDA/Ag hybrid surfaces of PDA/Ag/NH/wood and PDA/Ag/SH/ wood samples, respectively (Fig. 2d and e). These results were agreed with the report of AFM observation by Zhang et al. that indicated the changed roughness structure with immobilization of Ag on PDA layer. ${ }^{20}$ Therefore, it can be concluded that the modified surface topography and hierarchical structure determined the superhydrophobic characteristics.

FTIR spectra of the control and treated wood samples are shown in Fig. 3. For the control wood samples, the absorption band at $3427 \mathrm{~cm}^{-1}$ was assigned to the stretching vibration of the $\mathrm{OH}$ groups, and the band at $2900 \mathrm{~cm}^{-1}$ was assigned to $\mathrm{C}-\mathrm{H}$ stretching vibrations. The prominent band at $1740 \mathrm{~cm}^{-1}$ was assigned to the $\mathrm{C}=\mathrm{O}$ stretching of the acetyl groups, the bands at $1593 \mathrm{~cm}^{-1}$ and $1505 \mathrm{~cm}^{-1}$ were assigned to the aromatic skeletal vibration of lignin, and the band at $1240 \mathrm{~cm}^{-1}$ was assigned to $\mathrm{C}-\mathrm{O}$ stretching of the guaiacyl ring. ${ }^{21}$ Based on previous research, ${ }^{22,23}$ the absorbance peaks of PDA at 1510, 1600 , and $1274 \mathrm{~cm}^{-1}$ were assigned to the $\mathrm{N}-\mathrm{H}$ scissoring vibrations, stretching from the indole ring, and $\mathrm{C}-\mathrm{O}$ stretching from phenolic moieties, respectively. Interestingly, the characteristic peaks of PDA appeared similar to the peaks of the wood component lignin, likely due to the similitude between PDA molecule structure and the lignin structural unit. For the wood samples coated with PDA layers, the characteristic peak at 3427 $\mathrm{cm}^{-1}$ for the $\mathrm{OH}$ groups shifted to $3392 \mathrm{~cm}^{-1}$, probably due to

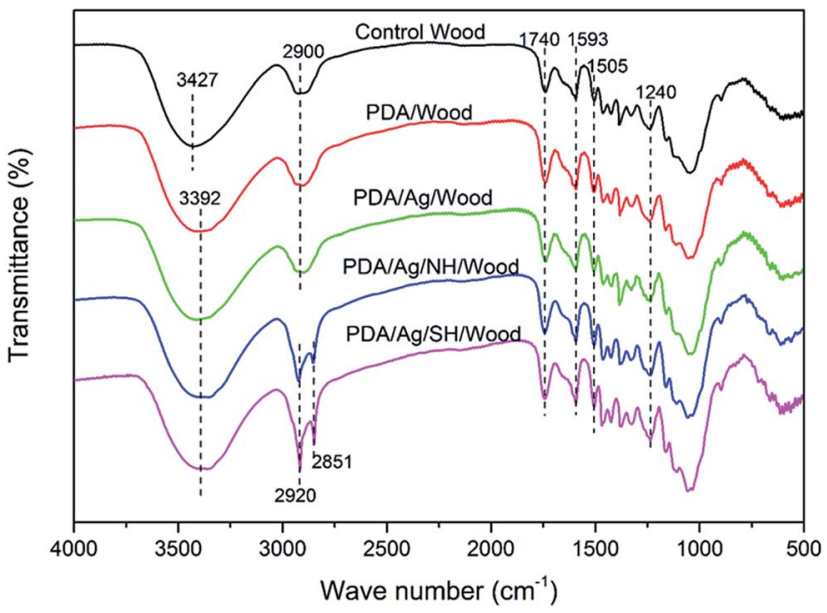

Fig. 3 FTIR spectrum of control wood, PDA/wood, PDA/Ag/wood, $\mathrm{PDA} / \mathrm{Ag} / \mathrm{NH} /$ wood and PDA/Ag/SH/wood.

the hydrogen bond formed between the wood hydroxyl groups and PDA. ${ }^{24}$ The two strong adsorption peaks at 2920 and 2851 $\mathrm{cm}^{-1}$ for the PDA/Ag/NH/wood and PDA/Ag/SH/wood samples were attributed to $-\mathrm{CH}_{3}$ and $-\mathrm{CH}_{2}$ asymmetrical stretching vibrations and symmetrical stretching vibrations from long alkyl chains, respectively. ${ }^{12}$ These results indicate that the PDA layer was tightly coated to the wood surfaces and that longchain hydrophobic groups were successfully grafted onto the PDA layer.

To better understand the surface chemical/elemental states of the superhydrophobic wood surface during each step of the preparation process, XPS analysis was conducted as presented in Fig. 4. As shown in Fig. 4a, the control wood only showed $\mathrm{C}$ and $\mathrm{O}$ signals, whereas PDA/wood had $\mathrm{C}, \mathrm{N}$, and $\mathrm{O}$ signals from the PDA layer. As expected, the characteristic Ag peaks appeared in the spectrum, indicated that $\mathrm{Ag}$ nanoparticles and clusters were formed on the PDA-coated wood. When the PDA/Ag/wood samples were modified with octadecanethiol, S 2p signal was observed. Fig. 4b and c show the $\mathrm{C} 1 \mathrm{~s}$ core-level spectra of the control wood and PDA/wood. The $\mathrm{C}$ 1s core-level spectrum of (a)
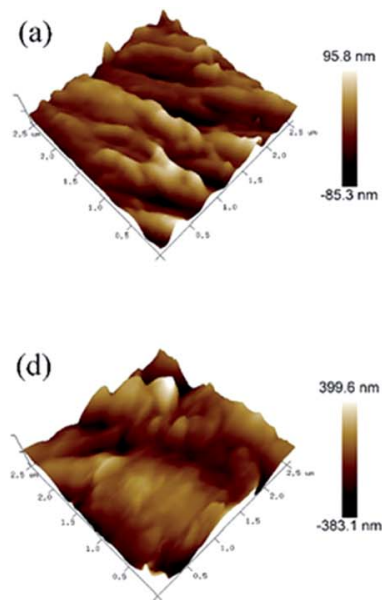

(b)
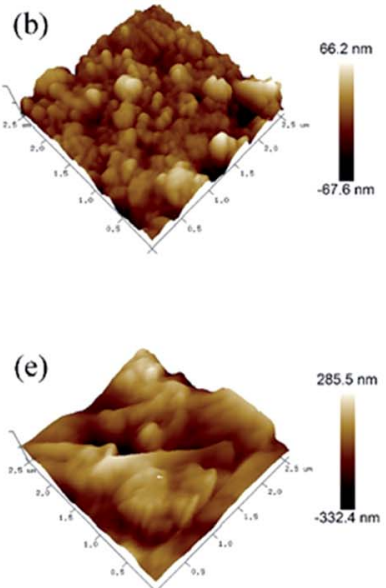

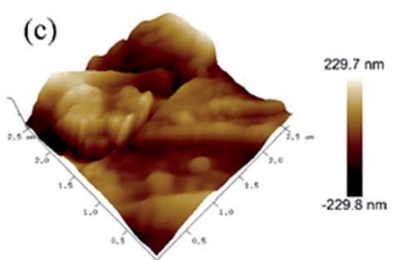

Fig. 2 AFM 3D topography of (a) control wood, (b) PDA/wood, (c) PDA/Ag/wood, (d) PDA/Ag/NH/wood, and (e) PDA/Ag/SH/wood. 


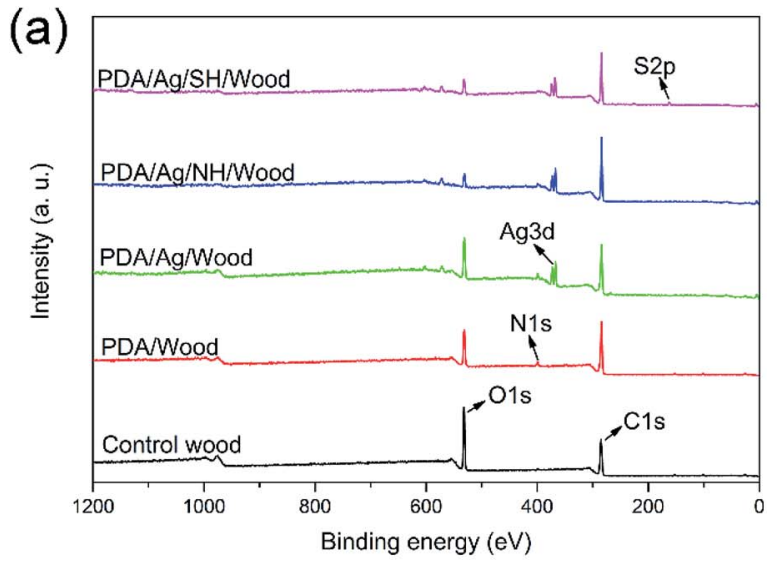

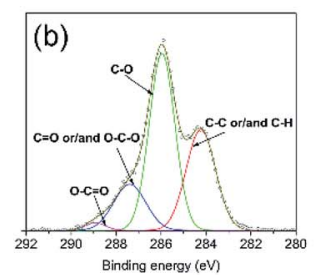
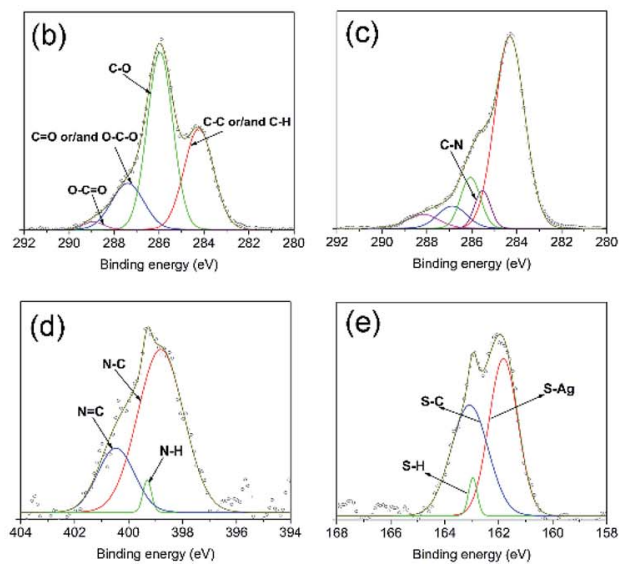

Fig. 4 XPS wide-scan (a), C 1s core-level spectra of (b) control wood and (c) PDA/wood, N 1s core-level spectra of (d) PDA/Ag/NH/wood, and S $2 p$ core-level spectra of (e) PDA/Ag/SH/wood.

control wood could be curve-fitted with four peak components, with binding energies at $284.2 \mathrm{eV}$ for $\mathrm{C}-\mathrm{C}$ or/and $\mathrm{C}-\mathrm{H}$ species, at $285.9 \mathrm{eV}$ for $\mathrm{C}-\mathrm{O}$ species, at $287.4 \mathrm{eV}$ for $\mathrm{C}=\mathrm{O}$ or/and $\mathrm{O}-\mathrm{C}-\mathrm{O}$ species, and at $288.9 \mathrm{eV}$ for $\mathrm{O}-\mathrm{C}=\mathrm{O}$ species. ${ }^{25}$ After the PDA coating treatment, the $\mathrm{C}$ 1s core-level spectrum of PDA/wood changed to five peak components, and the additional peak at $285.5 \mathrm{eV}$ was likely attributable to the $\mathrm{C}-\mathrm{N}$ species. ${ }^{26}$ Fig. $4 \mathrm{~d}$ shows the $\mathrm{N} 1$ s core-level spectra of $\mathrm{PDA} / \mathrm{Ag} / \mathrm{NH} /$ wood, which can be curved-fitted with three peak components, one at $398.8 \mathrm{eV}$ for the $\mathrm{N}-\mathrm{C}$ species, one at $399.3 \mathrm{eV}$ for the $\mathrm{N}-\mathrm{H}$ species, and another at $400.5 \mathrm{eV}$ for the $\mathrm{N}=\mathrm{C}$ species, ${ }^{27}$ as predicted in Scheme 1c. Fig. 4e shows the $\mathrm{S} 2 \mathrm{p}$ core-level spectra of $\mathrm{PDA} / \mathrm{Ag} / \mathrm{SH} / \mathrm{wood}$, and the $\mathrm{S} 2 \mathrm{p}$ spectrum can be curved-fitted with three peaks, corresponding to $\mathrm{S}-\mathrm{Ag}$ species at $161.8 \mathrm{eV}$, $\mathrm{S}-\mathrm{H}$ species at $162.8 \mathrm{eV}$, and $\mathrm{S}-\mathrm{C}$ species at $163.3 \mathrm{eV},{ }^{28,29}$ indicating the formation of a $\mathrm{C}-\mathrm{S}$ bond between the sulfur atom of octadecanethiol and the PDA layer and a $\mathrm{Ag}-\mathrm{S}$ bond between the $\mathrm{Ag}$ nanoparticles and clusters and the sulfur atom. ${ }^{28} \mathrm{Combining}$ the FT-IR analysis, these results demonstrated that the long chain alkyl groups successfully grafted onto the prepared rough surfaces via the previously hypothesized Michael addition or Schiff base reactions.

\section{Superhydrophobic property}

Fig. 5 illustrates the changes of CAs on the longitudinal surface (Fig. 5a) and transverse surface (Fig. 5b) over time. The CAs on different surfaces of the control wood, PDA/wood and PDA/Ag/ wood decreased rapidly over a short time. In contrast, the CAs on the longitudinal and transverse surfaces of $\mathrm{PDA} / \mathrm{Ag} / \mathrm{NH} /$ wood and PDA/Ag/SH/wood showed no obvious change and all remained larger than $150^{\circ}$ at $180 \mathrm{~s}$, indicating excellent superhydrophobic properties. Different wood surfaces possess distinguishing morphologies (Fig. 1). The longitudinal surface of wood displayed a micro-grooved structure, and the transverse surface showed a honeycomb-like appearance. In our previous work, after the direct grafting of long-chain alkyl groups to wood cell walls, the CAs on the transverse surface of treated wood samples were larger than $150^{\circ}$, but only about $140^{\circ}$ on the longitudinal surface, and the reasons were analyzed according to the model of Cassie and Baxter. ${ }^{12,30}$ Applying the musselinspired dopamine chemistry method, the modified wood surfaces all exhibited outstanding superhydrophobic properties.

As shown in Fig. 6a, the blue-dyed spherical water droplets rested on the PDA/Ag/NH/wood longitudinal surface. Fig. $6 \mathrm{~b}$ shows the spherical water droplets rolling down the slope with
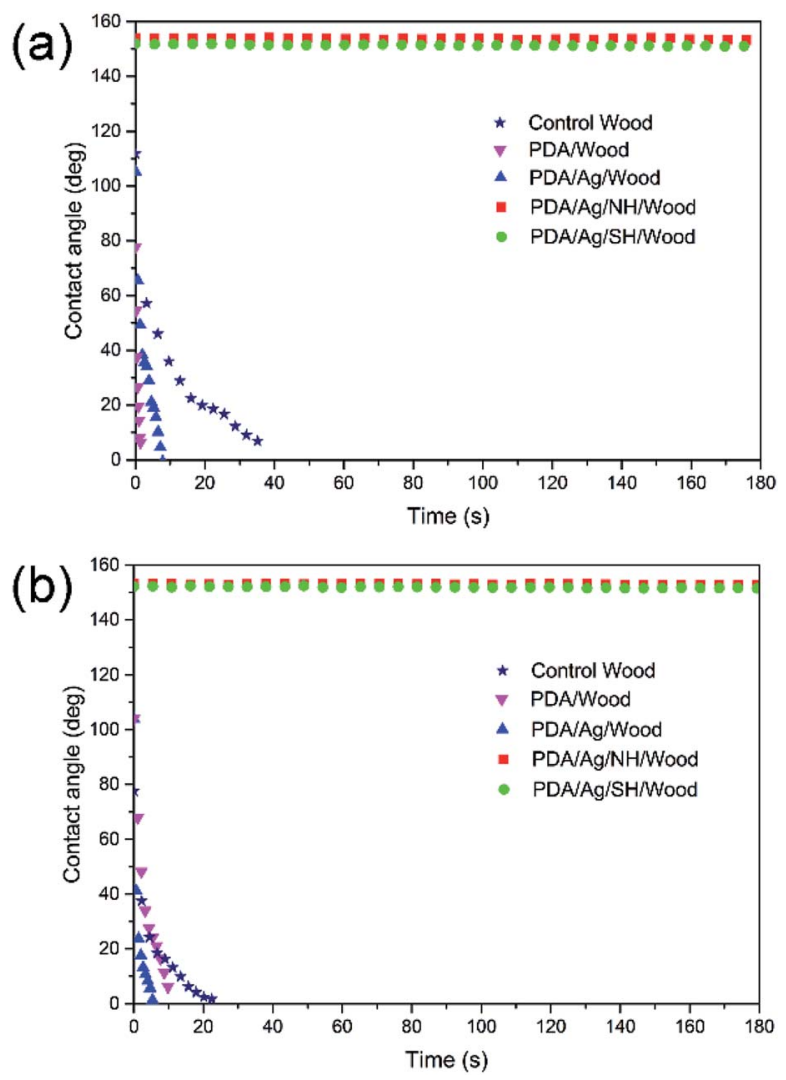

Fig. 5 Contact angle as a function of time for control wood, PDA/ wood, PDA/Ag/wood, PDA/Ag/NH/wood, and PDA/Ag/SH/wood on (a) longitudinal surface and (b) transverse surface. 


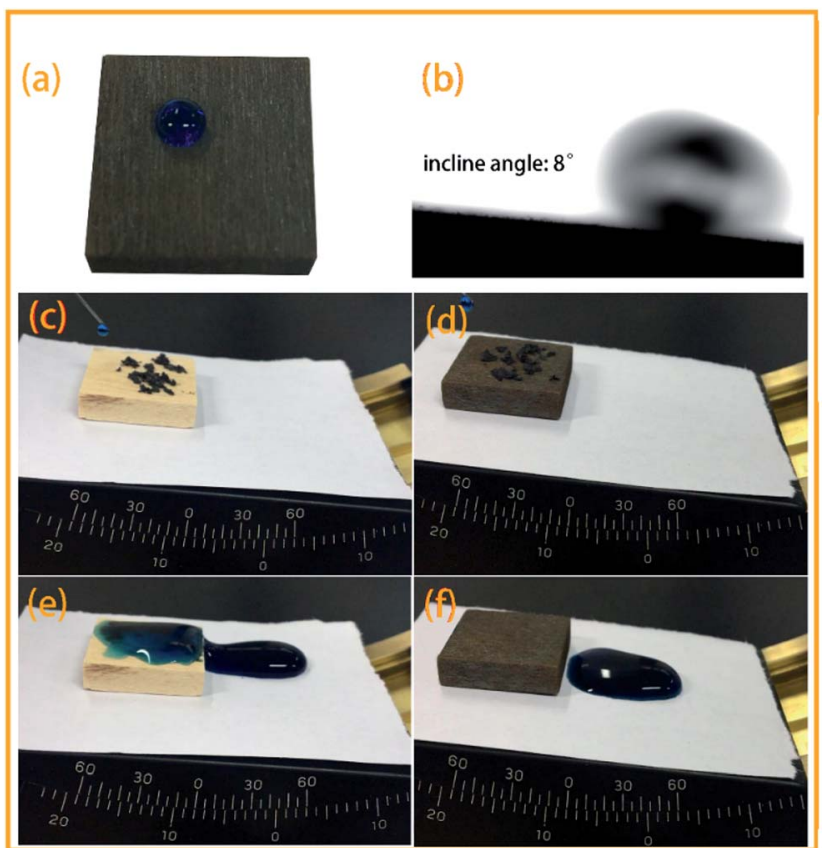

Fig. 6 Optical photographs of dyed water droplets on (a) PDA/Ag/NH/ wood; (b) profile of water droplets on the $8^{\circ}$ incline surface; (c and e) snapshots of the self-cleaning process on control wood surfaces; $(d$ and f) snapshots of the self-cleaning process on PDA/Ag/NH/wood surfaces.

a $8^{\circ}$ incline angle. The self-cleaning property of the PDA/Ag/NH/ wood surface was characterized by a dirt-removal test, in which water is dropped onto wood surface that is contaminated with carbon powder. When dropped onto the slightly titled $\left(8^{\circ}\right)$ wood surface, the water droplets merged with the carbon powder and stuck to the control wood surface, leaving a dirty and wet surface (Fig. 6c and e). In contrast, the water droplets readily rolled off the $\mathrm{PDA} / \mathrm{Ag} / \mathrm{NH} /$ wood surface, removing the dirt and resulting in a clean and dry surface (Fig. $6 \mathrm{~d}$ and $\mathrm{f}$ ).

The PDA/Ag/NH/wood surface exhibited a mirror-like phenomena when observed at an oblique angle (Fig. 7a), which is a typical characteristic of superhydrophobic surface. The superhydrophobic surface trapped air, and formed solidliquid-air interfaces that can effectively maintain a dry surface under water. Fig. 7b and c show the CAs and SAs of the PDA/Ag/ $\mathrm{NH} /$ wood samples after immersion in different chemical reagents (separate solutions of $\mathrm{HCl}, \mathrm{pH}=2, \mathrm{NaOH}, \mathrm{pH}=12, n$ hexane, acetone, ethanol, and DMF) for $24 \mathrm{~h}$, and $100{ }^{\circ} \mathrm{C}$ boiled water for $2 \mathrm{~h}$. The values of CAs were all above $150^{\circ}$ and the SAs were all below $10^{\circ}$, indicating that the superhydrophobic samples exhibited excellent stability and durability in harsh conditions. We next characterized the observed CA changes of $\mathrm{PDA} / \mathrm{Ag} / \mathrm{NH} /$ wood and PDA/Ag/SH/wood surfaces after ultrasonic washing $(40 \mathrm{kHz}, 100 \mathrm{~W})$ for $60 \mathrm{~min}$, as shown in Fig. 7d. The CAs of the wood surface all remained above $150^{\circ}$ after ultrasonic washing, demonstrating the excellent adhesive property of the PDA layer. Fig. 7e shows that the superhydrophobicity is stable under long-time UV light. The CAs of the surface remained above $150^{\circ}$ after $168 \mathrm{~h}$ of UV radiation, demonstrating the outstanding ability of the modified surfaces to withstand UV radiation. Fig. $7 f$ shows the surface repellency toward the three common household liquids (cola, orange juice, and milk) on the PDA/Ag/NH/wood samples surfaces. Similar to the water droplets, the liquid droplets remained steadily spherical on the superhydrophobic wood surface (the CAs were

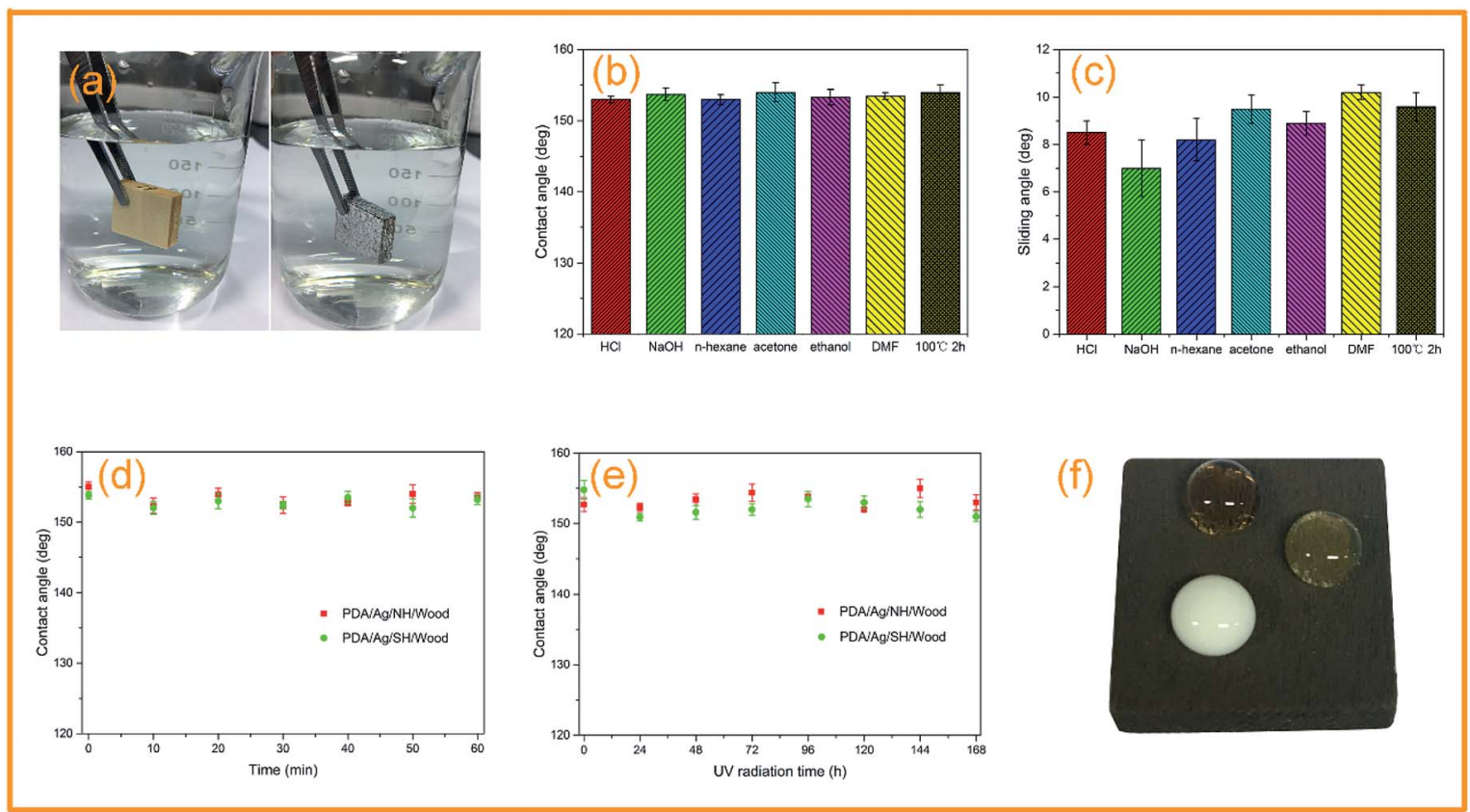

Fig. 7 Physical and chemical properties characterizations. (a) Mirror-like phenomenon, the left is control wood and the right is PDA/Ag/NH/ wood; (b) CAs for PDA/Ag/NH/wood samples soaked in different chemical solutions; (c) SAs for PDA/Ag/NH/wood samples soaked in different chemical solutions; (d) CAs as a function of time of ultrasonic washing in water for the treated wood surfaces; (e) CAs as a function of UV radiation exposure time for the treated wood surfaces; (f) commonhousehold liquids on the superhydrophobic wood surface. 
all about $150^{\circ}$ ). These results can demonstrate that the asprepared bionic superhydrophobic surfaces have great durability under harsh conditions.

Many superhydrophobic wood surfaces have been prepared, however, the poor environment durability limited its practical application. Some studies have been reported that the CAs gradually decreased on as-prepared superhydrophobic wood surfaces with subjecting to various harsh conditions, such as ultrasonic washing, strong acid/base or organic solvents immersion, etc. ${ }^{31-33}$ However, in the present study, the mussel-inspired functionalization method can form a stable and durable superhydrophobic coating on wood surfaces, which possess excellent performance against various harsh environments.

\section{Microstructure, oil-absorption capacity and oil-water separation test}

Fig. 8 shows the SEM images of the pristine sponge and the $\mathrm{PDA} / \mathrm{Ag} / \mathrm{NH} / \mathrm{sponge}$ at different magnification. As shown in

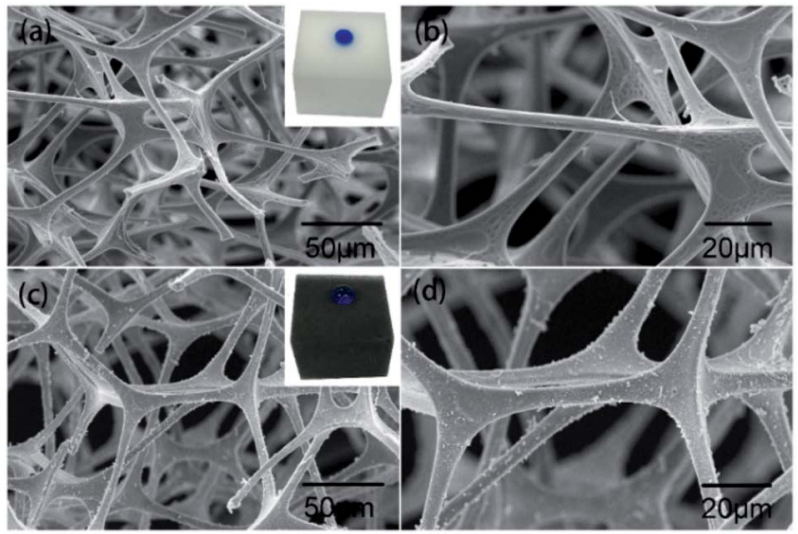

Fig. 8 FE-SEM observations of ( $a$ and $b$ ) control sponge, and ( $c$ and $d$ ) $\mathrm{PDA} / \mathrm{Ag} / \mathrm{NH} /$ sponge at different magnifications.
Fig. 8a and b, the pristine sponge exhibits an interconnected porous structure with smooth surfaces, and the inserted image in Fig. 8a shows blue-dyed water permeating into the pristine sponge. A large number of PDA and Ag nanoparticles and clusters are uniformly immobilized onto the sponge skeleton after treatment (Fig. 8c and d), and the inserted image in Fig. 8c shows the spherical water droplet resting steadily on the surface of as-prepared sponge with a contact angle of about $154^{\circ}$. The synergistic effect of intrinsic porous structure and micro/nano roughness contributes to the superhydrophobicity of sponge.

The produced PDA/Ag/NH/sponge has superhydrophobicity, high porosity, and light mass, and the absorption ability is the important parameter determining its practical use for oil absorption. Chloroform and $n$-hexane were used to demonstrate the absorption process, as illustrated in Fig. 9a and b. When the PDA/Ag/NH/sponge was placed in contact with chloroform or $n$-hexane (dyed with oil red) either under the water bottom or on water surface, the chloroform or $n$-hexane was absorbed within a few seconds, leaving clear water. To further evaluate the oils/organic solvents removal ability, absorption experiments of some representative pollutants in daily life/ industry were tested next, including $n$-hexane, dodecane, dichloromethane, trichloromethane, diethylether, styrene, xylene, sunflower oil and pump oil. As detailed in Fig. 9c, the $\mathrm{PDA} / \mathrm{Ag} / \mathrm{NH} /$ sponge showed excellent absorption abilities for these pollutants, with absorption capacity that ranged from 73 to 156 times the sponge weight. The observed differences in absorption capacity are likely due to the densities of the oils/ organic solvents used. In addition, the recyclability and reusability of a material are also key criterions for its effective use in practical oil cleanup. Here we evaluate the ability of this material to be recycled and reused though an absorption-squeeze cycle of $n$-hexane (Fig. 9d). After 20 absorption/squeezing cycles, the absorption capacity of the PDA/Ag/NH/sponge decreased only slightly, to $92.7 \%$ of its initial absorption capacity, showing

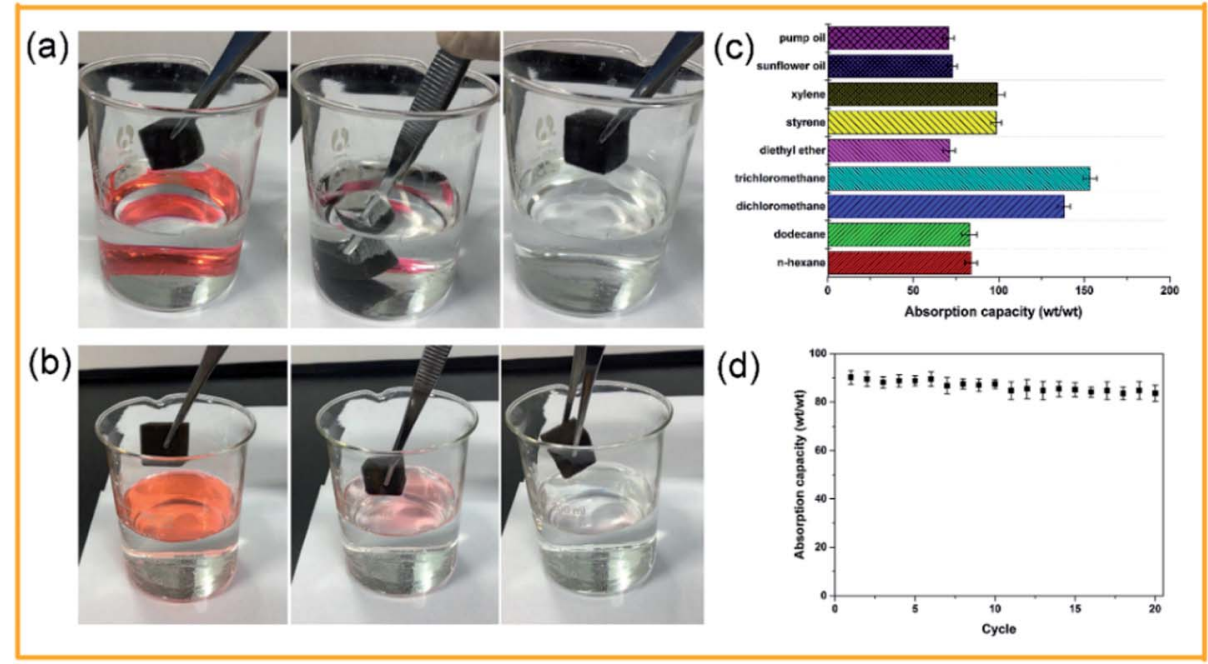

Fig. 9 The removal process. (a) Removal of chloroform (stained with oil red) from the water surface; (b) removal of $n$-hexane (stained with oil red) from the bottom of the water using the PDA/Ag/NH/sponge; (c) absorption capacities of the oils/organic solvents for the PDA/Ag/ $\mathrm{NH} /$ sponge; and (d) recyclability of the PDA/Ag/NH/sponge in $n$-hexane through the squeezing method. 
a great potential application in oil spill treatment. These results coincided with many prepared superhydrophobic and superoleophilic melamine sponges in many literatures which possessed high absorption capacity and recyclability for a wide range of organic compounds. ${ }^{34,35}$ Especially, Wang et al. prepared durable superhydrophobic/superoleophilic polyurethane sponges with the similar mussel-inspired method, which can selectively remove organic pollutants from water with a high separation efficiency. ${ }^{36}$

Fig. 10 shows the SEM images of the pristine stainless steel mesh and the $\mathrm{PDA} / \mathrm{Ag} / \mathrm{NH} / \mathrm{mesh}$ at different magnification levels. As shown in Fig. 10a and b, imaging of the pristine stainless steel mesh made of single-layered cross-knitted steel wires showed a smooth surface with an approximate pore diameter of $50 \mu \mathrm{m}$. The inset image in Fig. 10a shows the bluedyed water spreading and permeating into the pristine mesh. Imaging of the modified material revealed many PDA and Ag nanoparticles and clusters that were uniformly immobilized onto the surface of the stainless steel mesh. These particles on the surface form micro/nano roughness on the pristine porous structure, which creates a superhydrophobic mesh with a contact angle of about $152^{\circ}$ (inset image in Fig. 10c).

The PDA/Ag/NH/mesh is a thin, superhydrophobic, and porous material that is potentially suitable as a filter for oil/ water separation. Fig. 11 shows the process of oil/water separation. A mixture of water and trichloromethane (dyed with oil

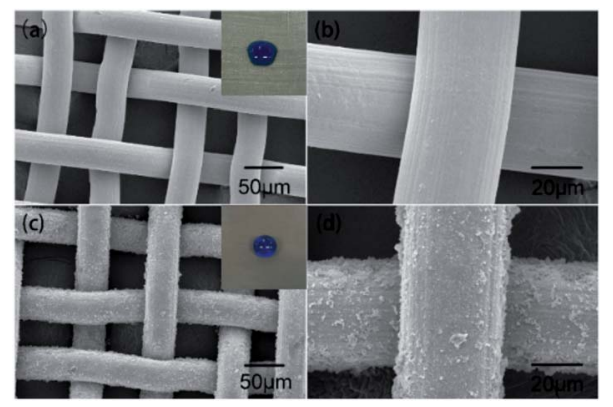

Fig. 10 FE-SEM observations of ( $a$ and $b$ ) control stainless steel mesh, and ( $\mathrm{c}$ and $\mathrm{d}$ ) PDA/Ag/NH/mesh at different magnifications.

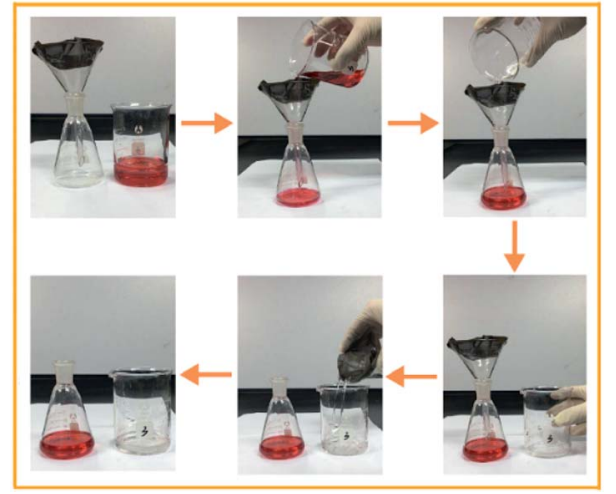

Fig. 11 Visual observation of the oil/water separation process by the $\mathrm{PDA} / \mathrm{Ag} / \mathrm{NH} /$ mesh. red) was poured onto the $\mathrm{PDA} / \mathrm{Ag} / \mathrm{NH} / \mathrm{mesh}$, and with only the driving force of gravity, trichloromethane passed through the mesh easily and rapidly, and flowed into the underneath conical flask. The water was repelled by the superhydrophobic mesh, remained inside the concave mesh, and then was poured out of the glass beaker. The entire oil/water separation process can be accomplished with a short time and without the need for additional force. And the flux of this PDA $/ \mathrm{Ag} / \mathrm{NH} / \mathrm{mesh}$ is about $37.53 \mathrm{~L} \mathrm{~m}^{-2} \mathrm{~s}^{-1}$. The outstanding oil/water separation capacity was also reported in the previous literature using different methods like dip-coating, spray-coating, etc. ${ }^{37,38}$ And the similar mussel-inspired method was applied to prepare superwetting double-layer polyester materials for effective removal of both insoluble oils and soluble dyes in water by Wang et al. ${ }^{39}$ These studies may allow for a wide range of potential applications, e.g., oil/water separation, and wastewater cleaning, etc.

\section{Conclusions}

In summary, a facile and versatile method based on bionic mussel to prepare a superhydrophobic surface on porous materials (wood, sponge, and stainless stain mesh were tested here) was developed. The as-prepared superhydrophobic wood surfaces showed excellent durability under harsh environments, including strong acid, strong base, organic solvent, high-temperature boiling, ultrasonic washing, and ultraviolet aging. The obtained superhydrophobic sponge had excellent absorption capacity of 73-156 times that of the unmodified material for a series of oils/organic solvents and also demonstrated good recyclability. The superhydrophobic stainless steel mesh also showed outstanding oil-water separation ability, and has great potential applicability for environmental remediation. This approach is efficient, easy, and mild, with nonspecific substrate and surface demands, allowing a wide range of potential applications in biomimetic materials.

\section{Acknowledgements}

This research was supported by Key Laboratory of Bio-based Material Science and Technology (Northeast Forestry University), Ministry of Education (SWZCL 2016-11) and "The Fundamental Research Funds for the Central Universities" (No. 2016ZCQ01).

\section{Notes and references}

1 A. R. Studart, Chem. Soc. Rev., 2016, 45, 359-376.

2 J. T. Simpson, S. R. Hunter and T. Aytug, Rep. Prog. Phys, 2015, 78, 086501.

3 K. Nakayama, E. Tsuji, Y. Aoki, S. G. Park and H. Habazaki, J. Phys. Chem. C, 2015, 120, 15684-15690.

4 S. A. Khan, U. Zulfiqar, S. Z. Hussain, U. Zaheer, I. Hussain, S. W. Husain and T. Subhani, J. Sol-Gel Sci. Technol., 2017, 81, 912-920.

5 R. Sukamanchi, D. Mathew and K. S. S. Kumar, ACS Sustainable Chem. Eng., 2017, 5, 252-260. 
6 A. Costa, N. V. Dencheva, S. G. Caridade, Z. Z. Denchev and J. F. Mano, Adv. Mater. Interfaces, 2016, 3, 1600074.

7 Y. Wu, S. Jia, Y. Qing, S. Luo and M. Liu, J. Mater. Chem. A, 2016, 4, 14111-14121.

8 S. Huang, Y. Zhang, J. Shi and W. Huang, ACS Sustainable Chem. Eng., 2016, 4, 676-681.

9 H. Lee, S. M. Dellatore, W. M. Miller and P. B. Messersmith, science, 2007, 318, 426-430.

10 H. Zhu, W. Luo, P. N. Ciesielski, Z. Fang, J. Zhu, G. Henriksson, M. E. Himmel and L. Hu, Chem. Rev., 2016, 116, 9305-9374.

11 Q. Sun, Y. Lu and Y. Liu, J. Mater. Sci., 2011, 46, 7706-7712.

12 K. Wang, Y. Dong, Y. Yan, C. Qi, S. Zhang and J. Li, RSC Adv., 2016, 6, 98248-98256.

13 L. Xie, Z. Tang, L. Jiang, V. Breedveld and D. W. Hess, Surf. Coat. Technol., 2015, 281, 125-132.

14 D. Su, C. Huang, Y. Hu, Q. Jiang, L. Zhang and Y. Zhu, Appl. Surf. Sci., 2011, 258, 928-934.

15 M. Ma, Y. Mao, M. Gupta, K. K. Gleason and G. C. Rutledge, Macromolecules, 2005, 38, 9742-9748.

16 M. Liu, Y. Qing, Y. Wu, J. Liang and S. Luo, Appl. Surf. Sci., 2015, 330, 332-338.

17 M. Sasaki, N. Kieda, K. Katayama, K. Takeda and A. Nakajima, J. Mater. Sci., 2004, 39, 3717-3722.

18 A. Nakajima, K. Hashimoto, T. Watanabe, K. Takai, G. Yamauchi and A. Fujishima, Langmuir, 2000, 16, 7044-7047.

19 Z. Xu, K. Miyazaki and T. Hori, Appl. Surf. Sci., 2016, 370, 243-251.

20 L. Zhang, Z. Liu, Y. Wang, R. Xie, X. J. Ju, W. Wang, L. G. Lin and L. Y. Chu, Chem. Eng. J., 2017, 309, 691-699.

21 Y. Dong, Y. Yan, S. Zhang, J. Li and J. Wang, Eur. J. Wood Wood Prod., 2015, 73, 457-464.

22 S. Kang, M. Baginska, S. R. White and N. R. Sottos, ACS Appl. Mater. Interfaces, 2015, 7, 10952-10956.

23 R. Luo, L. Tang, J. Wang, Y. Zhao, Q. Tu, Y. Weng, R. Shen and N. Huang, Colloids Surf., B, 2013, 106, 66-73.
24 H. J. Salavagione, G. Martínez and M. A. Gómez, J. Mater. Chem., 2009, 19, 5027-5032.

25 P. N. Diouf, T. Stevanovic, A. Cloutier, C. H. Fang, P. Blanchet, A. Koubaa and N. Mariotti, Appl. Surf. Sci., 2011, 257, 3558-3564.

26 R. Sa, Z. Wei, Y. Yan, L. Wang, W. Wang, L. Zhang, N. Ning and M. Tian, Compos. Sci. Technol., 2015, 113, 54-62.

27 S. Chen, Y. Cao and J. Feng, ACS Appl. Mater. Interfaces, 2013, 6, 349-356.

28 D. Zang, F. Liu, M. Zhang, X. Niu, Z. Gao and C. Wang, Chem. Eng. J., 2015, 262, 210-216.

29 A. Vaish, D. J. Vanderah, L. J. Richter, M. Dimitriou, K. L. Steffens and M. L. Walker, Chem. Commun., 2015, 51, 6591-6594.

30 K. Wang, Y. Dong, Y. Yan, W. Zhang, C. Qi, C. Han, J. Li and S. Zhang, Wood Sci. Technol., 2017, 51, 395-411.

31 P. Wang, M. Du, M. Zhang, H. Zhu, S. Bao, M. Zou and T. Yang, Chem. Eng. J., 2014, 248, 307-314.

32 C. Liu, S. Wang, J. Shi and C. Wang, Appl. Surf. Sci., 2011, 258, 761-765.

33 P. Cai, N. Bai, L. Xu, C. Tan and Q. Li, Surf. Coat. Technol., 2015, 277, 262-269.

34 E. C. Cho, Y. S. Hsiao, K. C. Lee and J. H. Huang, RSC Adv., 2015, 5, 53741-53748.

35 Q. Liu, K. Meng, K. Ding and Y. Wang, ChemPlusChem, 2015, 80, 1435-1439.

36 B. Li, L. Li, L. Wu, J. Zhang and A. Wang, ChemPlusChem, 2014, 79, 850-856.

37 D. Deng, D. P. Prendergast, J. MacFarlane, R. Bagatin, F. Stellacci and P. M. Gschwend, ACS Appl. Mater. Interfaces, 2013, 5, 774-781.

38 L. Feng, Z. Zhang, Z. Mai, Y. Ma, B. Liu, L. Jiang and D. Zhu, Angew. Chem., Int. Ed., 2004, 43, 2012-2014.

39 B. Li, L. Wu, L. Li, S. Seeger, J. Zhang and A. Wang, ACS Appl. Mater. Interfaces, 2014, 6, 11581-11588. 